\title{
A Mathematical Model for Interpretable Clinical Decision Support with Applications in Gynecology
}

\author{
Vanya M.C.A. Van Belle ${ }^{1,2}$, Ben Van Calster ${ }^{1,2,3}$, Dirk Timmerman ${ }^{3}$, Tom Bourne ${ }^{3,4}$, Cecilia Bottomley $^{5}$, \\ Lil Valentin ${ }^{6}$, Patrick Neven ${ }^{7,8}$, Sabine Van Huffel ${ }^{1,2}$, Johan A.K. Suykens ${ }^{1,2}$, and Stephen Boyd ${ }^{9}$ \\ ${ }^{1}$ Department of Electrical Engineering (ESAT-SCD), Katholieke Universiteit Leuven,Kasteelpark Arenberg 10, \\ B-3001 Leuven, Belgium \\ 2 IBBT - Future Health Department, Katholieke Universiteit Leuven,Kasteelpark Arenberg 10, B-3001 Leuven, \\ Belgium \\ 3 Department of Development and Regeneration, University Hospitals Leuven, Herestraat 49, B-3000 Leuven, \\ Belgium \\ ${ }^{4}$ Department of Obstetrics and Gynaecology, Imperial College London, Hammersmith Campus, Du Cane Road, \\ London, W12 0HS, United Kingdom \\ ${ }^{5}$ Chelsea and Westminster Hospital, 369 Fulham Road, London, SW10 9NH, United Kingdom \\ 6 Department of Obstetrics and Gynecology, Skåne University Hospital Malmö, Lund University, SE20502 Malmö, \\ Sweden \\ 7 Multidisciplinary Breast Centre (MBC), University Hospitals Leuven,Herestraat 49, B-3000 Leuven, Belgium \\ 8 Department of Gynaecological Oncology, University Hospitals Leuven, Herestraat 49, B-3000 Leuven, Belgium \\ ${ }^{9}$ Department of Electrical Engineering Stanford, Stanford University, CA 94305-9510 USA
}

\section{Detailed description of the data}

\section{Diagnosis of the malignancy of adnexal masses}

The dataset contains information on 3,511 patients with at least one overt persistent adnexal mass who received surgery. The data were recruited in three phases. In phase 1 1,066 patients in 9 centers were recruited between 1999 and 2002. In phase 1b three of these centers included 507 extra patients from the period 2002 to 2005. In phase 2 1,938 patients were recruited from 2005 to 2007. Seven of the centers from phase 1 and 12 new centers contributed to the second phase. Overall, the patients were recruited in 21 centers in 9 countries. Patients were included if the principal investigator from one of the participating centers assessed the mass. In case of multiple masses, the information from the most complex mass was included. Patients who were pregnant, refused transvaginal ultrasonography or did not undergo surgical removal of the mass within 120 days of the ultrasound examination were excluded. The variables considered in the analysis are summarized in Table S1. The outcome is the diagnosis of a tumor as benign or malignant (including borderline, primary invasive and metastatic tumors). Out of the 3,511 included tumors, 951 (27\%) were malignant and 2,560 $(73 \%)$ were benign.

Table S1: Description of the variables of the adnexal mass data set considered in this work.

\begin{tabular}{llll}
\hline \multicolumn{1}{c}{ variable } & \multicolumn{1}{c}{ description } & unit/level & type \\
\hline age & age of patient & years & continuous \\
famhistovca & family members with history of ovarian cancer & binary (0 no; 1 yes) & binary \\
famhistbrca & nr of family members with history of breast cancer count & ordinal \\
pershistovca & personal history of ovarian cancer & binary (0 no; 1 yes) & binary \\
pershistbrca & personal history of breast cancer & binary (0 no; 1 yes) & binary \\
parity & nr of deliveries & count & ordinal \\
hysterectomy & hysterectomy & binary (0 no; 1 yes) & binary \\
hormtherapy & current use of hormonal therapy & binary (0 no; 1 yes) & binary \\
bilateral & masses on both sides & binary (0 no; 1 yes) & binary \\
\hline
\end{tabular}


Table S1 - continued from previous page

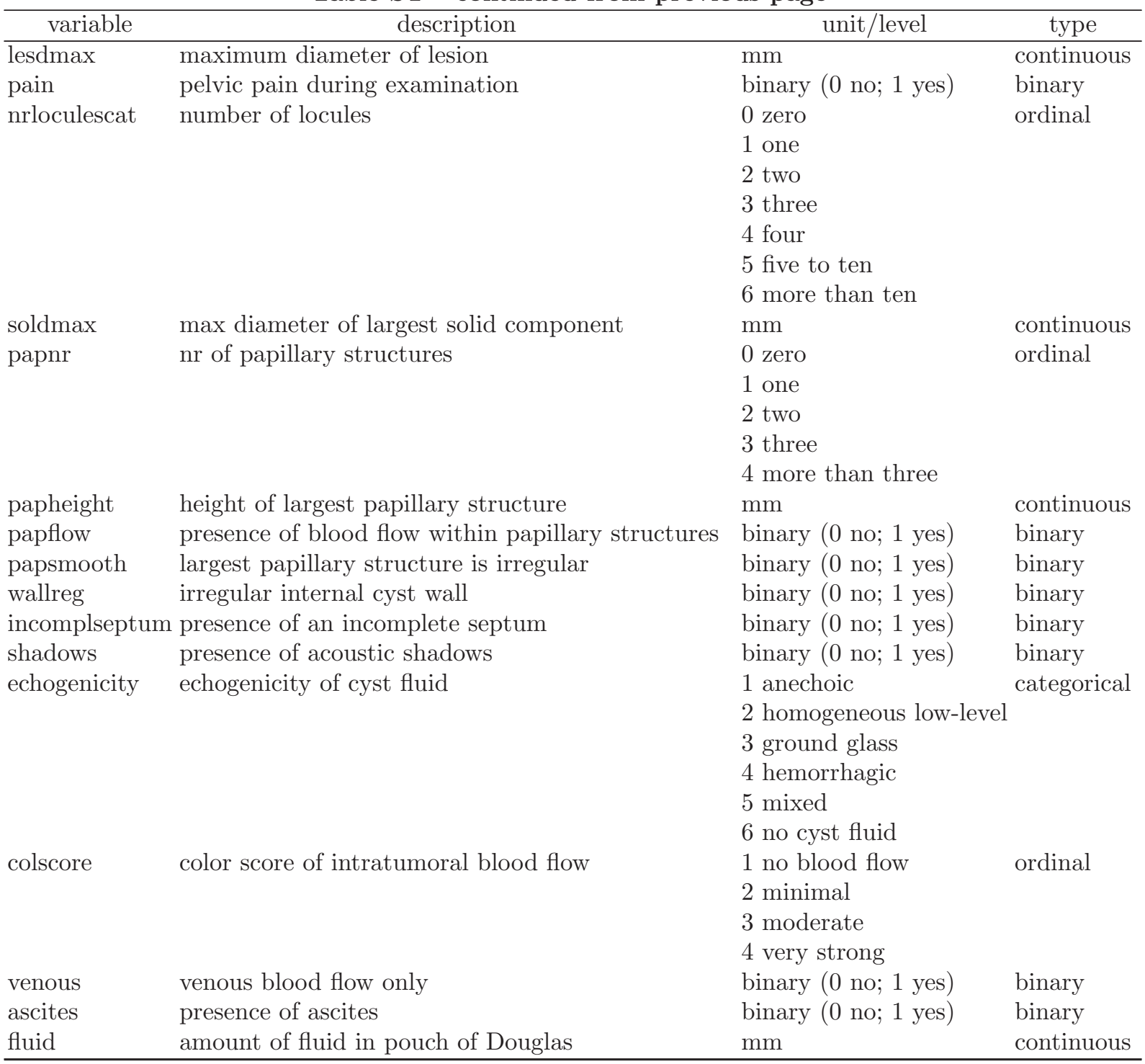

\section{Prediction of non-viability of pregnancies}

This data set is a prospective observational cohort of all women attending a single early pregnancy unit from January to October 2006. Inclusion criteria were: a positive pregnancy test and a gestational age of less than 12 weeks. Patients presenting with postpartum complications or complications from termination of pregnancy or recently diagnosed early pregnancy loss were not considered. Exclusion criteria were: multiple pregnancies, patients who appeared not to be pregnant and patients who underwent termination of pregnancy. Patients presenting with more than one pregnancy over the study period were included only once (first presenting pregnancy). A total of 1,435 pregnancies remained for analysis. The data were randomly divided into a training and test set, containing 955 and 480 patients, respectively. The outcome was defined as viable when a live fetus had been seen at the routine 11-14 week scan, performed after the initial scan. The outcome was non-viable whenever the pregnancy had resulted in a miscarriage, an ectopic pregnancy or a failed pregnancy of unknown location. Out of the 1,435 included pregnancies, 550 (38\%) were non-viable and $885(62 \%)$ were 
viable. The variables considered in the experiments are summarized in Table S2. Missing values were imputed using distance-aided selection of donors [1].

Table S2: Description of the variables of the pregnancy data set considered in this work.

\begin{tabular}{|c|c|c|c|}
\hline variable & description & unit/level & type \\
\hline age & age of patient & years & continuous \\
\hline \multirow[t]{5}{*}{ pbac } & bleeding score & 0: no bleeding & ordinal \\
\hline & & 1: light bleeding & \\
\hline & & 2: moderate bleeding & \\
\hline & & 3: heavy bleeding & \\
\hline & & 4: bleeding with cloths & \\
\hline gravida & number of pregnancies (including the present pregnancy) & & ordinal \\
\hline nodel 24 & number of deliveries after 24 weeks & & ordinal \\
\hline notop & number of termination of pregnancies & & ordinal \\
\hline noemis & number of early miscarriages & & ordinal \\
\hline \multirow[t]{2}{*}{ nopul } & previous pregnancies of unknown location & 0: no & binary \\
\hline & & 1: yes & \\
\hline \multirow[t]{2}{*}{ nolmis } & previous late miscarriages & 0: no & binary \\
\hline & & 1: yes & \\
\hline \multirow[t]{2}{*}{ noect } & previous ectopic pregnancies & 0: no & binary \\
\hline & & 1: yes & \\
\hline gestdates & gestational age: days since start of last menstrual period & days & continuous \\
\hline vas & pain score & $\mathrm{cm}$ & continuous \\
\hline \multirow[t]{2}{*}{ pain } & is the patient in pain? (unspecified) & $0:$ no & binary \\
\hline & & 1: yes & \\
\hline meangsd & mean gestational sac diameter & $\mathrm{mm}$ & continuous \\
\hline meanysd & mean yolk sac diameter & $\mathrm{mm}$ & continuous \\
\hline \multirow[t]{2}{*}{ fhrseen } & is a fetal heart rate seen? & 0: no & binary \\
\hline & & 1: yes & \\
\hline \multirow[t]{2}{*}{ fetus } & is a fetus seen? & 0: no & binary \\
\hline & & 1: yes & \\
\hline crl & crown-rump length & $\mathrm{mm}$ & continuous \\
\hline \multirow[t]{2}{*}{ regdates } & did the patient report a regular cycle of 26 to 30 days? & 0: no & binary \\
\hline & & 1: yes & \\
\hline
\end{tabular}

\section{Illustration of the use of a nomogram for ovarian cancer diagnosis}

Figure S1 is a nomogram, illustrating a logistic regression model for diagnosing adnexal masses as benign or malignant. This model was proposed in [2]. The nomogram consists of several rulers. The first one is a reference ruler, the last two are rulers to obtain the results. The rulers in between correspond to each relevant variable. Consider a 70- year-old woman with an adnexal mass, without ascites, with flow in the papillary structures of the mass, a maximal diameter of the solid component of $25 \mathrm{~mm}$, with an irregular cyst wall and no acoustic shadows. Looking at the ruler representing age, the patient will receive 61 points. The absence of ascites results in zero points. Due to the blood flow in the papillary structures, the patient receives 33 points. In a similar way 80 points are gained for the diameter of the solid component, 27 for the irregular cyst wall and 84 for the absence of shadows. In total, this patient receives 285 points. To obtain the risk of malignancy, a line needs to be drawn between the total points ruler at 285 points towards the risk of malignancy ruler. The patient specific risk of malignancy is nearly $90 \%$. 
points

age

presence of ascites

flow in papillations

diameter of solid component

irregular cyst wall

presence of acoustic shadows

total points

probability on a malignancy

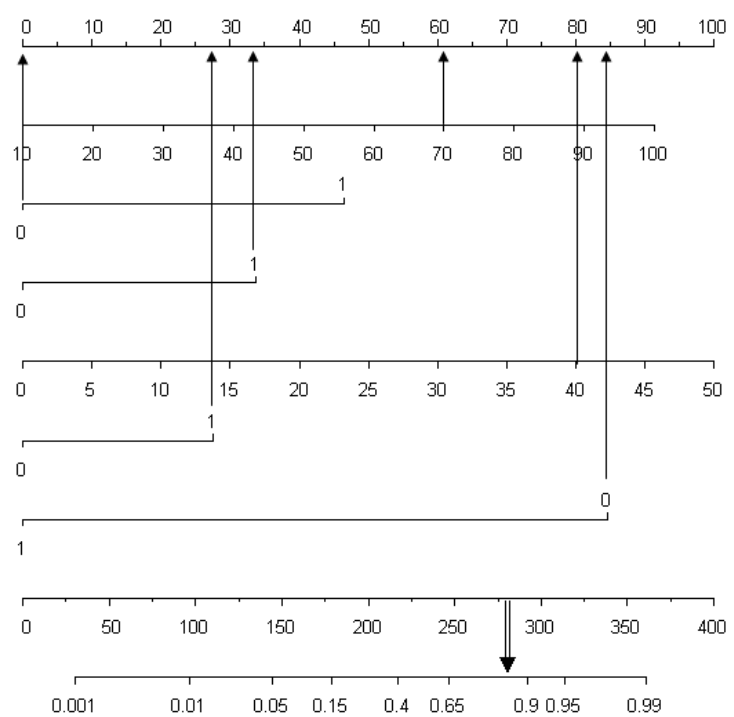

Fig. S1: Nomogram for the diagnosis of ovarian cancer derived from a logistic regression model (LR2 in [2]). For a paper-based implementation, the clinician has to draw vertical lines from the value of each variable to the point axis (upward arrows). The obtained points need to be added to get the total number of points for the patient. The risk of a malignant mass is found by drawing a vertical line from the total number of points to the risk of malignancy axis (see downward arrow from the total points axis to the risk axis).

\section{Illustration of a model implementation created with the ICS methodology}

This Section gives further explanation on the video in movie S1. This application was implemented in Microsoft Excel. It might be incorporated in software packages, touchscreen applications and included in clinical decision support systems. Starting from an empty screen, the user has to go to the data entry sheet and start the application by clicking the Click here to start the application button. An application sheet will pop up and the user can click on the intervals that apply to his/her patient. When more than one, or none of the intervals for a variable is marked, an error will appear on the screen. Only when all questions of Table 3 in the manuscript are answered, the user will receive a risk calculation after clicking the calculate risk button. A new window pops up indicating the total score and the corresponding risk. This window is closed by clicking the close result button. A click on the clear form button brings the user back to the initial Excel sheet. The results are stored in the ovarian sheet. For each variable, the allocated points are given and the total number of points is represented together with the associated risk. Other possible implementations are provided in movie S2 and movie S3.

\section{Software}

The proposed method was implemented as a convex optimization problem [3] in Matlab, using $\mathrm{CVX}^{\dagger}$.

\footnotetext{
* http://www.mathworks.com/products/matlab/

$\dagger$ http://cvxr.com/cvx/
} 


\section{References}

1. Siddique J, Harel O (2009) MIDAS: A sas macro for multiple imputation using distance-aided selection of donors. Journal of Statistical Software 29: 1-18.

2. Timmerman D, Testa AC, Bourne T, Ferrazzi E, Ameye L, et al. (2005) Logistic regression model to distinguish between the benign and malignant adnexal mass before surgery: a multicenter study by the international ovarian tumor analysis group. Journal of Clinical Oncology 23: 8794-8801.

3. Boyd S, Vandenberghe L (2004) Convex optimization. Cambridge University Press, Cambridge. 